\title{
Efficient Source Routing Scheme for Mobile Ad hoc Networks
}

\author{
R. Bhuvaneswari \\ Research Scholar \\ Anna University of Technology \\ Coimbatore
}

\author{
M. Viswanathan ,PhD \\ Senior Deputy director \\ Fluid Control Research Institute (FCRI) \\ Palakkad, Kerala
}

\begin{abstract}
Mobile Ad hoc Network (MANET) is a collection of mobile nodes without any centralized administration. These nodes collect the information through overhearing and store information in route caches through routing protocol. When the route cache freshness is absent, it leads to the stale route information resulting in pollution caches. If the node overhears the packet to another node, node's energy consumption occurs unnecessarily. The main goal of this research work is to reduce the effect of overhearing and avoid the stale route problems while improving the energy efficiency using the Efficient Source Routing Scheme (ESRS) algorithm. Due to the lack of route cache update, the stale route entry and overhearing is originated among the network. For that, we developed five mechanisms to improve route cache performance in DSR. By simulation results the proposed algorithm achieves better performance than the existing methods.
\end{abstract}

\section{General Terms}

Source Routing algorithm, Stale Route Problem and cache update.

\section{Keywords}

MANET, DSR, Stale route entry, Cache freshness, overhearing, route cache update and energy efficiency.

\section{INTRODUCTION}

Mobile Ad Hoc Network (MANET) is a selfconfiguring system of mobile routers linked by wireless links which consequently combine to form an arbitrary topology. Thus, the network's wireless topology may alter rapidly and unpredictably. However, due to the lack of any fixed infrastructure, it becomes complicated to exploit the present routing techniques for network services, and this provides some huge challenges in providing the security of the communication, which is not done effortlessly as the number of demands of network security conflict with the demands of mobile networks, largely due to the nature of the mobile devices .e.g. low power consumption, low processing load.

\section{DYNAMIC SOURCE ROUTING PROTOCOL AND THE EFFECTS OF OVERHEARING}

DSR is a reactive routing protocol which is able to manage a MANET without using periodic table-update messages like table-driven routing protocols do. DSR was specifically designed for use in multi-hop wireless ad hoc networks. Ad-hoc protocol allows the network to be completely self organizing and self-configuring which means that there is no need for an existing network infrastructure or administration. DSR allows the network to be completely selforganizing and self-configuring, without the need for any existing network infrastructure or administration. Dynamic Source Routing (DSR), is a reactive routing protocol that uses source routing to send packets. It uses source routing which means that the source must know the complete hop sequence to the destination.

Each node maintains a route cache, where all routes it knows are stored. The route discovery process is initiated only if the desired route cannot be found in the route cache. To limit the number of route requests propagated, a node processes the route request message only if it has not already received the message and its address is not present in the route record of the message. DSR uses source routing, i.e. the source determines the complete sequence of hops that each packet should traverse. This requires that the sequence of hops is included in each packet's header. A negative consequence of this is the routing overhead every packet has to carry. However, one big advantage is that intermediate nodes can learn routes from the source routes in the packets they receive. Since finding a route is generally a costly operation in terms of time, bandwidth and energy, this is a strong argument for using source routing. Another advantage of source routing is that it avoids the need for up-to-date routing information in the intermediate nodes through which the packets are forwarded since all necessary routing information is included in the packets. Finally, it avoids routing loops easily because the complete route is determined by a single node instead of making the decision hop-by-hop.

\subsection{Effects of Overhearing}

Overhearing [3] means a node picks up packets that are destined for other nodes. Wireless nodes will consume power unnecessarily due to overhearing transmissions of their neighboring nodes. Wireless nodes consume power unnecessarily due to overhearing the transmissions of their neighbors. This is often the case in a typical broadcast environment. For example, as the IEEE 802.11 wireless protocol defines, receivers remain on and monitor the common channel all the time. Thus the mobile nodes receive all packets that hit their receiver antenna. Such scheme results in significant power consumption because only a small number of the received packets are destined to the receiver or needed to be forwarded by the receiver.

\subsection{Problem of Stale route in source routing}

It is unconditional overhearing that dramatically exaggerates the problem. This is because DSR generates more than one RREP packets for a route discovery to offer alternative routes in addition to the primary route to the source. While the primary route is checked for its validity during data 
communication between the source and the destination, alternative routes may remain in route cache unchecked even after they become stale. This is the case not only for the nodes along the alternative routes, but also for all their neighbors because of unconditional overhearing.

\section{PREVIOUS WORK}

$\mathrm{Hu}$ C et.al [5] developed the 802.11 Power Saving Mode (PSM) applicable in multihop MANET with Dynamic Source Routing (DSR) protocol. The drawback in integrating the DSR protocol with 802.11 PSM comes from unnecessary or unintended overhearing and DSR depends on broadcast flood of control packets.

Lim S et.al [6] explored a mechanism called RandomCast mechanism. Here a node may decide not to overhear i.e. a unicast message and not to forward and a broadcast message when it receives an advertisement during an ATIM window, thereby reducing the energy cost without affecting the network performance. In addition to the energy consumption, overhearing brings in several undesirable consequences. It could aggravate the stale route problem, the main cause of which is node mobility.

Sree Ranga Raju [7] proposed a conservative approach to gather route information. It does not allow overhearing and eliminates existing route information using timeout. This necessitates more RREQ messages which in turn results in more control overheads in routing.

Laura marie feeney [8] analysed the energy consumption model for routing protocols in ad hoc networks. They have also shown that new insights are provided into costly protocol behaviours and suggests opportunities for improvement at the protocol and link layers.

Ramesh et.al [9] explored a new scheme called efficient energy management to achieve minimum energy consumption with the presence of overhearing. Here they have proposed five modules like networking module, packet division module, randomcast module and energy efficient balancing module in order to avoid redundant rebroadcasts and thus save additional energy.

Ashish K et.al [10] \& Charles E Perkins et.al [11] proposed the on-demand routing protocols DSR and AODV, before sending a packet to the destination, discovers a route. Route maintenance is invoked when node detects link failure. In order to avoid route discovery for each packet, on-demand routing protocols utilizes cache routes previously learnt.

Ashish Shukla [12] proposed a cache timeout policy to predict route cache lifetime, and to expunge stale route cache entries, which are timed out. Many techniques have been proposed for route cache organization and its effect on the performance of on-demand routing protocols. But the concentration of cache timeout policy is very less.

It is used in route cache implementation to prevent stale route from being used. So, a technique for reducing the unintended overhearing of neighboring nodes is done with the help of RandomCast mechanism and for prevention of stale route problem, a cross-layer cache timeout policy is implemented. Time out policy derives cache timeouts of individual links that are present in route cache by utilizing Received Signal Strength Indicator (RSSI) information. So to fulfil the objective and to overcome the drawbacks, a message overhearing and forwarding mechanism called RandomCast [4] is chosen which makes a judicious balance between energy and network performance.

Sangeetha et.al [13] used the prediction mechanism and smart prediction mechanism which performs better than EOLSR protocol and reduce traffic load. In MANET state information such as residual energy level plays an important role in route selection. If latest information is not collected by nodes, then performance may degrade. They have also evaluated the effect of time at which state information was collected in ideal and realistic approach and concluded that although ideal approach is better than realistic but increase in frequency of packets improve the performance very little and also increase traffic overhead.

B. H. Liu [14] used hello messages to distribute transmission power, and uses the minimum power required to connect to a neighbor, while considering the costs of reception of a packet at the neighboring nodes.

Freeny [15] suggests that if ATIM window is fixed then energy saving can be affected. DPSM improves this performance by using the variable ATIM window. It allows the sender and receiver node to change their ATIM window dynamically. The ATIM window size increased when some packets are pending after the current window is expired. The data packets carry the current length of the ATIM window and the nodes overhear this modify their ATIM window length. DPSM allows the sender and receiver node to switch of their radio immediately after their transmission is over. The energy saving performance of DPSM is better as compare to IEEE 802.11 DCF in term of power saving however it is computationally more complex.

S.Singh and C.S.Raghavendra [16] projected energy efficiency technique which is achieved by using two separate channels, one for control and other for data. RTS/CTS signals are transmitted over the control channel while data are transmitted over data channel. Nodes with packet to transmit sends a RTS over the control channel, and waits for CTS, if no CTS they receives within a specific time then node enters to a backoff state. However, if CTS is received, then the node transmits the data packet over the data channel. The receiving node transmits a busy tone over the control channel for its neighbours indicating that its data channel is busy. The use of control channel allows nodes to determine when and how long to power off. The length of power off time is determined by different condition. After waking up, a node access the channel over the data channel and found multiple transmission going on. The node uses a probe protocol in this case to find how much time it will power off. Simulation results shows that good range of power saving is achieved.

In IEEE 802.11[17] standard protocols, it has two types of power managements. First type is known as power save (PS) mode for infrastructure based wireless network and the second type is known as IBSS Power Saving mode, which is for infrastructure-less networks. In the former method nodes in PS mode consume less power compare to active mode operation. The access point buffered the Media Authentication Code (MAC) service date unit (MSDU) and transmits them at designated time by the help of Traffic Indication Map (TIM) and delayed traffic indication map (DTIM). This type of power saving mechanism is not suitable for ad hoc network environment as there is no central coordinator like access point. On the other hand IBSS PS mode is applicable to fully connected single hop network where all the nodes are in the radio range to each other. Synchronized beacon interval is established by the node which initiates the IBSS and is maintained in a distributed fashion. All the nodes wake at the beginning of the beacon interval and wake till the end of the traffic window. The nodes participating in the traffic announcement remain awake till the end of beacon interval and the non-participator goes to sleep to conserve energy at the end of the traffic window. The amount of energy conserve by a node depends upon the time spent in the sleep state which can be affected by the state transition from sleep to active mode operation. The energy saving performance also 
depends upon the network size as well as on the length of the ATIM window and beacon interval duration.

The paper is organized as follows. The Section 1 describes introduction about MANET, overview of DSR protocol and stale route problems in DSR. Section 3 deals with the previous work which is related to the energy consumption. Section 4 is devoted for the implementation of source initiated energy efficient algorithm. Section 5 describes the performance analysis and the last section 6 concludes the work.

\section{IMPLEMENTATION OF DYNAMIC SOURCE ROUTING ALGORITHM}

In our proposed technique, we have used following mechanisms in order to avoid stale route problems, achieve minimum energy consumption. Dynamic Source Routing aggressively uses route caching. Using source routing, it is possible to cache every overheard route without causing loops. If any forwarding node caches any source route in a packet, it forwards the packets for possible future use. The destination node replies to all requests. Thus the source node learns many exchange routes to the destination nodes that are cached. Swap routes are useful in case the primary route breaks. If any intermediate node on a route learns routes to the source and destination as well as other intermediate nodes on that route. A large amount of routing information is gathered and cached with just a single query reply cycle. So these cached routes may be used in replying to subsequent route queries. The reply from caches provides dual performance advantages. First, it reduces route discovery latency and without replies from caches the route query flood will reach all nodes in the network. Cached replies satisfy the query flood early, thus saving on routing overheads.

If without an effective mechanism, stale cache entries are removed. Then, route replies may carry stale routes. Attempted data transmissions using stale routes incur overheads and generate additional error packets and can potentially pollute other caches when a packet with a stale route is forwarded or snooped on. In the following, there are three problems are identified with the DSR protocol that are the root cause of the stale cache problem.

Case $i$ :

If link breaks and route errors are not propagated to all caches that has an entry with the broken link. Instead, the RERR (Route error) is unicast only to the source whose data packet is accountable for identifying the link crack via a link layer feedback. We take only a limited number of caches are cleaned. The failure information is propagated by piggybacking it onto the subsequent route requests from the source. If the route requests may not be propagated networkwide, many caches may remain unclean.

\section{Case ii:}

Still now there is no mechanism is proposed to expire stale routes. If not fresh, stale cache entries will stay forever in the cache.

Case iii:

There is no way to determine the freshness of any route information. For an example, even after a stale cache entry is erased by a route error, a subsequent "in-flight" data packet carrying the same stale route can put that entry right back in. This problem is mixed by liberal use of snooping. Stale routes are chosen up by any other node overhearing any transmission. Thus, cache "pollution" can propagate fairly quickly.

\subsection{Time based Expiration of Route}

If we recall that link breakage is detected only by a link layer feedback, when an attempted data transmission fails. Thus loss of a route will go undetected if there is no attempt to use this route. A more proactive clock-based approach will be able to fresh up such routes. A clock based approach is based on the hypothesis that routes are only valid for a specific amount of time_T(timeout period) from their last use. Each node in a cached route now has an associated timestamp of last use. This timestamp is updated each time the cached route or part thereof is "seen" in a unicast packet being forwarded by the

node. The main portions of cached routes unused in the past _interval are pruned. The advantage of this approach depends critically on the proper selection of the timeout period A very small value for the timeout may cause many unnecessary route invalidations, while a very large value may defeat the purpose of this technique. Although well-chosen static values can be obtained for a given network, a single timeout for all the nodes may not be appropriate in all scenarios and for all network sizes. Therefore, a dynamic mechanism is desirable that allows each node to choose timeout values independently based on its observed route stability. The proposed technique heuristic approach for adaptive selection of timeouts locally at each node based on the average route lifetime and the time between links breaks seen by the node. When a cached route breaks due to link breakage or upon receipt of a route error, the lifetime of the broken route is computed as the time elapsed since it was last entered in the cache. Average route lifetime is obtained using the lifetimes of all broken routes in the past. Time of latest link breakage seen by a node is also maintained.

When route breaks occur uniformly in time, average route lifetime itself provides a good estimate. However when many route breaks occur in short bursts with a large separation in time, the average route lifetime does not accurately predict during the periods of no route breaks. The value of route life time _Tis computed periodically and is used to expire stale entries from the cache. In the experiments, _every half a second and route cache is computed then checked for stale entries.

\subsection{Energy Consumption Model}

All the discussions in this section and the following sections correspond to a mobile graph $U_{M}=U_{I} U_{2} \ldots U_{T}$ generated for an source-destination $(s-d)$ session by sampling the network topology at instants of packet origination $t s_{1}, t s_{2}, \ldots, t s_{T}$. Let $P k=v_{0} v_{1} \ldots v p$ be the static $s-d$ path in $\mathrm{U}_{i}=\left(\mathrm{R}_{i}, \mathrm{O}_{i}\right)$ at time $t s i$. Here, $v_{0}=$ source and $v_{l}=$ destination and $\left(v p_{-1}, v p\right) \in \mathrm{O}_{i}$ for $p$ $=1,2, \ldots, l$ are the hops of the $s-d$ path. All the energy consumption calculations for the $s-d$ path at time tsi are strictly based on the snapshot of the network topology $\mathrm{U} i$ at $t s i$. Thus the queuing delays and propagation delays are neglected while assuming infinite channels. The packets are being instantaneously transmitted from source $s$ to destination d.

The energy consumed for a node to node traffic on the sourcedestination path $P i$ is modelled as the sum of the energy consumed along each hop. The energy consumption is modelled per hop considering complete overhearing (nondestination nodes receive the entire data packet), a reduced overhearing case where the non-destination nodes discard the data packet after scanning its header and when there is no overhearing.

The over hearing costs are presented at the non-destination nodes for each of the three following cases: 
$P t o p \_T x(v j-1)=$

Transenergy $\left(\frac{\text { Sizeof } \operatorname{Re} q T o S e n d+\text { Sizeoforiginaldata }+P D R}{\text { Bandwidth }}\right)$

Receenergy $*\left(\frac{\text { SizeofCleartosend }+ \text { Acksize }+ \text { RERR }}{\text { Bandwidth }}\right)$

Ptop_Rec(vj-1) =

Transenergy $\left(\frac{\text { SizeofClearToSend }+ \text { ACKsize }+ \text { RERR }}{\text { Bandwidth }}\right)$

$+$

Re ceenergy $*\left(\frac{\text { Sizeof } \operatorname{Re} \text { qtosend }+ \text { Datasize }+ \text { PDR }}{\text { Bandwidth }}\right)$

\subsubsection{Absolute overhearing}

Here the nodes are operating in promiscuous mode. The non-destination nodes at the neighborhood of the sender $v_{p-1}$ are stimulated for receiving the entire data packet. Including these nodes are charged for receiving the Request To Send (RTS) packet. Likewise, the non-destination nodes at the neighborhood of the receiver $v_{p}$ are charged for receiving the Acknowledge (ACK) and Clear To Send (CTS) packets.

$\forall l \in \operatorname{Nei}{ }_{-} \operatorname{Nodes}\left(v_{p-1}, t_{s i}\right), \operatorname{AOvhe}\left(l, v_{p-1}, t_{s i}\right)$ $=$

$$
\begin{aligned}
& \operatorname{Receenergy} *\left(\frac{\text { Sizeof } \operatorname{Re} \text { qtosend }+ \text { Datasize }+P D R}{\text { Bandwidth }}\right) \text { (3) } \\
& \forall h \in \text { Nei_Nodes }\left(v_{p}, t_{s i}\right), \text { AOvhe }\left(h, v_{p-1}, v_{p}, t_{s i}\right) \\
& =
\end{aligned}
$$$$
\text { Receenergy } *\left(\frac{\text { SizeofClearToSend }+ \text { ACKsize }+R E R R}{\text { Bandwidth }}\right)
$$

\subsubsection{Decreased overhearing}

Instead of the receiving the entire data packet, a node could scan only the header of the data packet and discard the remaining of the packet. Thus, the non-destination nodes at the neighbourhood of the sender $v_{p-1}$ are excited for only receiving the data packet header and the Request To Send packet. On the converse, the non-destination nodes at the neighborhood of the receiver $v j$ are charged for receiving the ACK and CTS packets. This simple strategy can bring significant power savings when the data size is considerably larger than the size of the header preceding the actual data in the data packet.

$\forall \quad l \in \operatorname{Nei}{ }_{-} \operatorname{Nodes}\left(v_{p-1}, \quad t_{s i}\right), \quad$ AOvhe $\left(l, \quad v_{p-1}, \quad t_{s i}\right)=$ Receenergy* $\left(\frac{\text { PSizeof Re qtosend }+ \text { Data }_{-} \text {Neigh_size }+ \text { PDrop }}{\text { Bandwidth }}\right)$

\subsubsection{Absence of Overhearing}

If node enters the snooze or sleep state when there is an ongoing transmission in its neighborhood in which the node is neither a transmitter nor a receiver. If an intended receiver of the data packet is assumed to be notified by the sender through energy-efficient IEEE 802.11 ATIM frame mechanism [11]. Nodes are assumed to be identified their neighbors through the beacon frames exchanged as part of the power saving mechanism. The energy consumed for the transmission and reception of the ATIM and beacon frames is assumed negligible. Such an assumption may not be completely true because when the topology changes more frequently, power saving strategies require nodes to be awake at least half of the beacon interval. On the other hand, the maximum energy savings are evaluated that could be obtained when the cost of overhearing is totally discarded.

$\forall l \in \operatorname{Nei}{ }_{-} \operatorname{Nodes}\left(v_{p-1}, t_{s i}\right)$, AOvhe $\left(l, v_{p-1}, t_{s i}\right)=0$

$\forall h \in \operatorname{Nei}{ }_{-} \operatorname{Nodes}\left(v_{p}, t_{s i}\right), \operatorname{AOvhe}\left(h, v_{p-1, l} v_{p}, t_{s i}\right)=0$

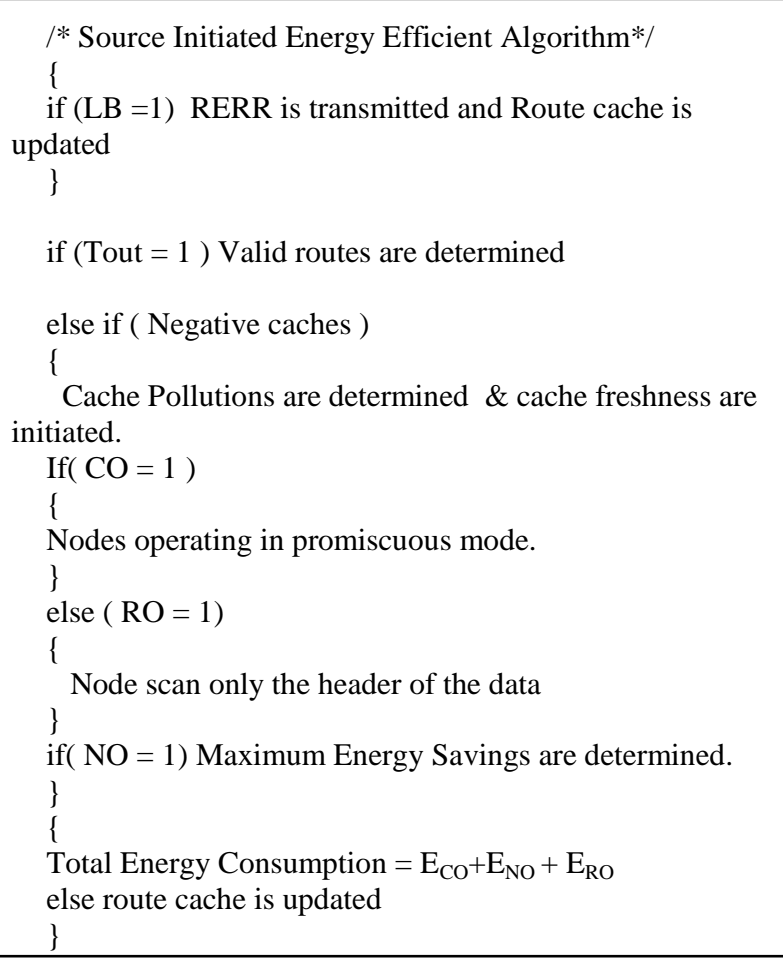

\section{PERFORMANCE ANALYSIS}

We use NS2 to simulate our proposed algorithm. In our simulation, 101 mobile nodes move in a 1000 meter $\times 1000$ meter square region for 50 seconds simulation time. All nodes have the same transmission range of 100 meters. The simulated traffic is Constant Bit Rate (CBR). Our simulation settings and parameters are summarized in table 1

Table.1. Simulation settings and parameters of ESRS

\begin{tabular}{|l|l|}
\hline No. of Nodes & 100 \\
\hline Area Size & 1000 X 1000 \\
\hline Mac & 802.11 \\
\hline Radio Range & $250 \mathrm{~m}$ \\
\hline Simulation Time & $60 \mathrm{sec}$ \\
\hline Traffic Source & CBR \\
\hline Packet Size & 512 bytes \\
\hline Mobility Model & Random Way Point \\
\hline
\end{tabular}

\subsection{Performance Metrics}

We evaluate mainly the performance according to the following metrics.

Control overhead: The control overhead is defined as the total number of routing control packets normalized by the total number of received data packets.

End-to-end delay: The end-to-end-delay is averaged over all surviving data packets from the sources to the destinations.

Packet Delivery Ratio: It is the ratio of the number .of packets received successfully and the total number of packets transmitted. 
The simulation results are presented in the next part. We compare our proposed algorithm with DBEE - CLA [18] and RANDOMCAST in presence of overhearing environment.

Figure 3 shows the results of average end-to-end delay for varying the nodes from 20 to 100 . From the results, we can see that ESRS scheme has slightly lower delay than the RANDOMCAST and DBEE-CLA scheme because of authentication routines.

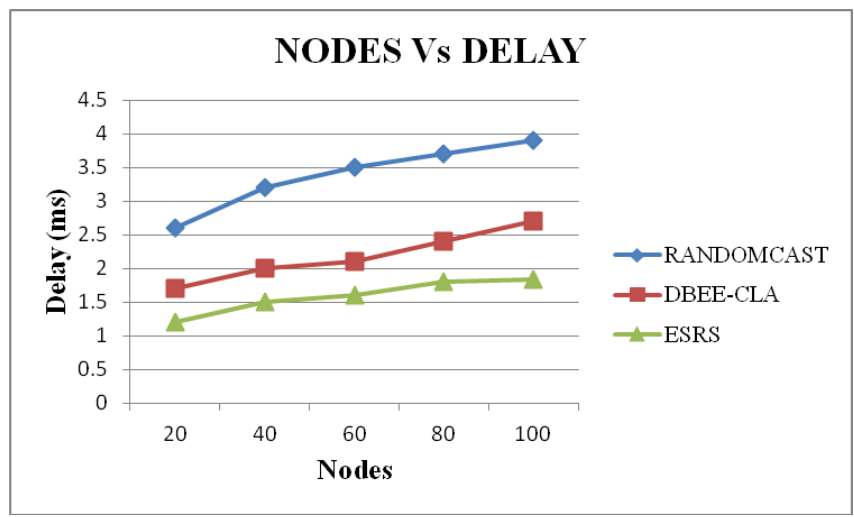

Fig. 3. Nodes Vs End to end Delay

Fig. 4, presents the energy consumption. The Comparison of energy consumption for ESRS, DBEE-CLA, RandomCast. It is clearly seen that energy consumed by ESRS is less compared to RandomCast and DBEE-CLA.

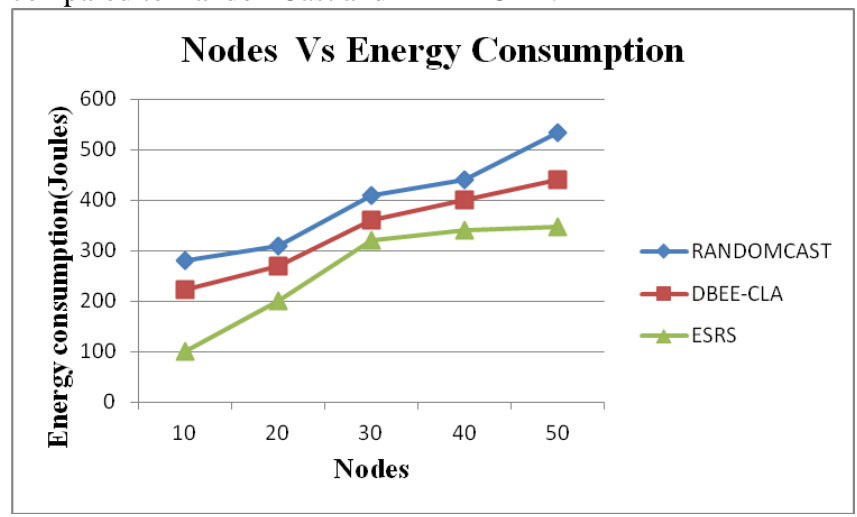

Fig. 4. No.of Nodes Vs Energy Consumption

Fig. 5, presents the comparison of overhead. It is clearly shown that the overhead of ESRS has low overhead than the RandomCast and DBEE-CLA.

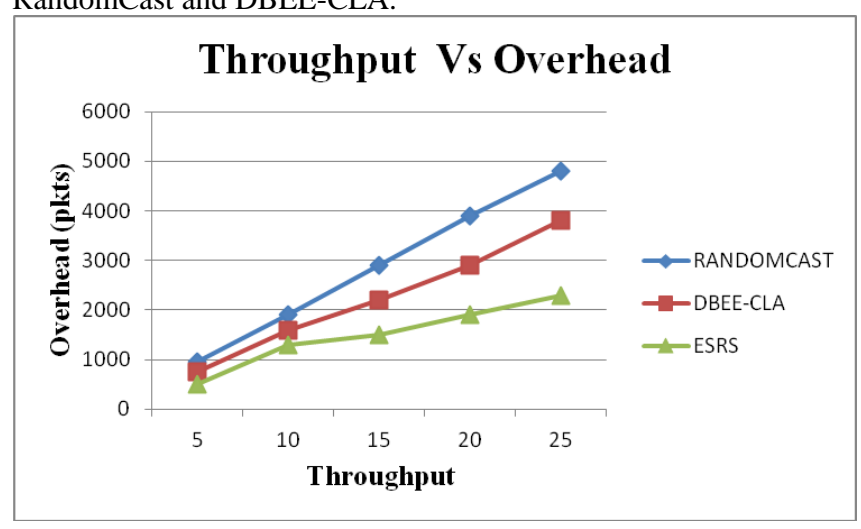

Fig. 5. Throughput Vs Overhead

Figure 6 shows the results of Mobility Vs Delay. From the results, we can see that ESRS scheme has slightly lower delay than the RANDOMCAST and DBEE-CLA scheme because of authentication routines.

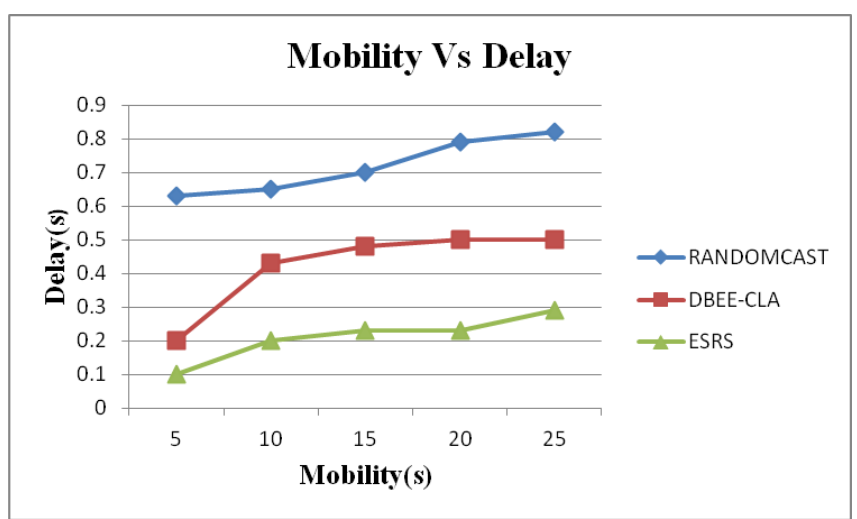

Fig. 6. Mobility Vs Delay

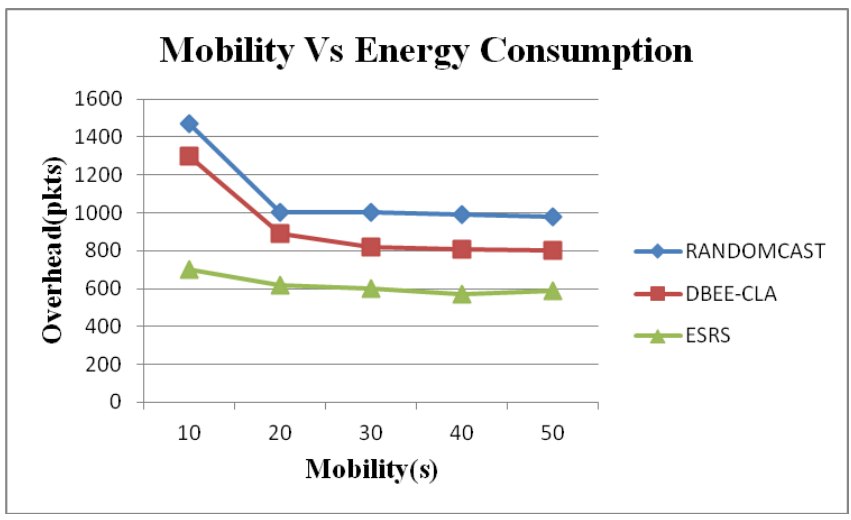

Fig.7.Mobility Vs Energy Consumption

Fig. 7, presents the comparison of total energy consumption while varying the mobility from 10 to 50 . It is clearly shown that the energy consumption of ESRS has low overhead than the RandomCast and DBEE-CLA.

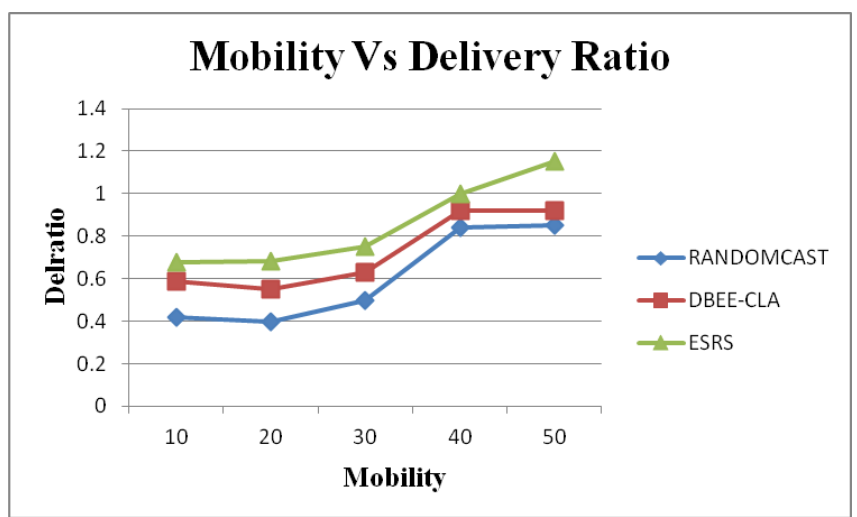

Fig.8. Mobility Vs Packet Delivery Ratio

Figure 8 show the results of average packet delivery ratio for the mobility $10,20 \ldots 50$ for the 100 nodes scenario. Clearly our ESRS scheme achieves more delivery ratio than the Randomcast and DBEE-CLA scheme since it has both reliability and security features.

\section{CONCLUSION}

In MANET, mobile nodes are moving randomly without any centralized administration. Due to that, the node consumes more energy unnecessarily. In this paper, we have developed a efficient source routing scheme with energy consumption 
model which attains minimum energy consumption to the mobile nodes. In the first phase of the scheme, route cache update and stale route avoidance is achieved using ESRS algorithm. In second phase, minimum energy consumption is achieved using energy consumption model. It uses three factors called utility factor, energy factor, mobility factor to favor packet forwarding by maintaining minimum energy consumption for each node. We have demonstrated the energy estimation of each node. By simulation results we have shown that the ESRS achieves good packet delivery ratio while attaining low delay, overhead, minimum energy consumption than the existing schemes Randomcast and DBEE-CLA while varying the number of nodes, node velocity and mobility.

\section{REFERENCES}

[1] Perkins C. Ad Hoc Networking: Addison-Wesley: 2001; $1-28$.

[2] David B. Johnson, David A. Maltz and Yih-Chun Hu, "The Dynamic Source Routing Protocol for Mobile Ad Hoc Networks (DSR)," Internet Draft, draft- ietf-manetdsr-09.txt,15April2004.

[3] Fang Liu, Kai Xing, Xiuzhen Cheng, Shmuel Rotenstreich , "Energy-efficient MAC layer protocols in ad hoc networks" Resource Management in Wireless Networking, Kluwer Academic Publishers, 2004, pp.142.

[4] Lim S, Yu C and Das C, "Rcast: A Randomized Communication Scheme for Improving Energy Efficiency in Mobile Ad Hoc Networks," Proc. 25th IEEE Int'l Conf. Distributed Computing Systems, pp. 123-132, 2005

[5] Ashish K. Shukla and Neeraj Tyagi, "A New Route Maintenance in Dynamic Source Routing Protocol," International Symposium on Wireless Pervasive Computing, Phuket, 2006.

[6] Jung E and Vaidya N, "A Power Control MAC Protocol for Ad Hoc Networks," Proc. ACM MobiCom, pp. 3647, 2002.

[7] Kyasanur P, Choudhury R. and Gupta I, "Smart Gossip: An Adaptive Gossip-Based Broadcasting Service for Sensor Networks," Proc. Second IEEE Int'l Conf. Mobile Ad Hoc and Sensor Systems, pp. 91-100, 2006.

[8] Laura Marie Feeney, "An Energy Consumption Model for Performance Analysis of Routing Protocols for Mobile Ad Hoc Networks", Mobile Networks and Applications, 2001, pp.239-249.

[9] V.Ramesh et.al, "An Efficient Energy Management Scheme For Mobile Ad-hoc Networks", International Journal of Research and Reviews in Computer Science (IJRRCS) Vol. 1, No. 4, December 2010, pp.173-176.

[10] Ashish K. Shukla and Neeraj Tyagi, "A New Route Maintenance in Dynamic Source Routing Protocol," International Symposium on Wireless Pervasive Computing, Phuket, 2006.

[11] Charles E. Perkins and Elizabeth M. Royer, "Ad-Hoc On-Demand Distance Vector Routing," In Second 1EEE Workshop on Mobile Computing Systems and Applications, pages 90-100, February 1999.
[12] Ashish Shukla, "Ensuring Cache Freshness in Ondemand Routing Protocols for Mobile Ad Hoc Network: A Cross-layer Framework "IEEE CCNC, 2007.

[13] Sangeetha et.al, "Energy Efficient Routing In MANET Using OLSR", International Journal on Computer Science and Engineering (IJCSE), Vol. 3 No. 4 Apr 2011, pp.1418-1421.

[14] B. H. Liu, Y. Gao, C. T. Chou and S. Jha, "An Energy Efficient Select Optimal Neighbor Protocol for Wireless Ad Hoc Networks," Technical Report, UNSW-CSE-TR0431, Network Research Laboratory, University of New South Wales, Sydney, Australia, October 2004.

[15] L.M. Freeny, -Energy efficient communication in ad hoc networks, $\|$ Mobile Ad Hoc Networking, Wiley-IEEE press, pp. 301-328, 2004.

[16] S.Singh and C.S.Raghavendra, $\|$ PAMAS power aware multi-access protocol with signaling for ad hoc networks, $\|$ ACM Computer Communication Review, vol 28(3), pp.5-26, July 1998.

[17] Sofiane Boukli Hacene and Ahmed Lehireche, "Coherent Route Cache In Dynamic Source Routing For Ad Hoc Networks", Computer Science Journal of Moldova, vol.19, no.3(57), 2011, pp.304-319.

[18] R.Bhuvaneswari \& Dr.M.Viswanathan, “ Demand Based Effecive Energy Utilization in Mobile Ad Hoc Networks", International Journal of Computer Science Issues, Vol.9, Issue2, No.2,March 2012, pp.439-445.

\section{AUTHORS PROFILE}

M.Viswanathan. Senior Deputy Director in Fluid Control Research Institute (FCRI), a Public Sector Undertaking under Government of India, Palakkad, Kerala, India. He obtained graduate degree in Electronics and Communication Engineering from the University of Madras, Madras, India, received his Master's Degree in Electronics Engineering from IIT Kanpur and Doctoral Degree in Electronics from Bharathiar University, Coimbatore, India. He has 25 years of vast experience and had undergone training at NEL, UK in the area of flow measurement and B\&K, Denmark in the area of Noise and Vibration. He has published many Technical papers in International Journals and presented papers in conferences held in India and abroad.

R.Bhuvaneswari has received the B.E. Degree in Electronics and Communication Engineering from Govt. College of Technology, Bharathiar University, Coimbatore, Tamil Nadu, India in 1989, M.E.Degree in Applied Electronics from the Govt. College of Technology, Bharathiar University, Coimbatore, Tamil Nadu, India in 1994 and she is currently pursuing Ph.D. degree in Electronics and Communication Engineering from the Anna University of Technology Coimbatore Coimbatore, Tamil Nadu, India. Her areas of interests include Communication networks, Wireless Communication, Mobile Communication, Digital Communication, Biomedical Applications, Applied Electronics, Computer Networks, Mobile Adhoc Networks (WiFi, WiMax, HighSlot GSM) \& Network Security. She has also presented recently two papers on Mobile Adhoc Networks in conferences held in India. 